\title{
Parameter Dependence of Stable Invariant Manifolds for Delay Differential Equations under $(\mu, \nu)$-Dichotomies
}

\author{
Lijun Pan \\ School of Mathematics, Jia Ying University, Meizhou, Guangdong 514015, China \\ Correspondence should be addressed to Lijun Pan; plj1977@126.com
}

Received 20 May 2014; Accepted 14 September 2014; Published 27 October 2014

Academic Editor: Alfred Peris

Copyright (C) 2014 Lijun Pan. This is an open access article distributed under the Creative Commons Attribution License, which permits unrestricted use, distribution, and reproduction in any medium, provided the original work is properly cited.

We obtain the existence of stable invariant manifolds for the nonlinear equation $x^{\prime}=L(t) x_{t}+f\left(t, x_{t}, \lambda\right)$ provided that the linear delay equation $x^{\prime}=L(t) x_{t}$ admits a nonuniform $(\mu, \nu)$-dichotomy and $f$ is a sufficiently small Lipschitz perturbation. We show that the stable invariant manifolds are dependent on parameter $\lambda$. Namely, the stable invariant manifolds are Lipschitz in the parameter $\lambda$. In addition, we also show that nonuniform $(\mu, \nu)$-contraction persists under sufficiently small nonlinear perturbations.

\section{Introduction}

The theory of exponential dichotomy is attracting much attention in recent years. This is mostly because the concept of exponential dichotomy plays an important role in the obtention of invariant manifolds of differential equations. The notion of exponential dichotomy can be traced back to Perron in [1]. Since then, Sacker and Sell [2-5] investigated sufficient conditions for the existence of an exponential dichotomy, also in the infinite-dimensional setting. In [6], Barreira and Valls discussed the much weaker notion of nonuniform exponential dichotomy. This theory of nonuniform hyperbolicity was introduced in the seventies by Barreira and Pesin [7]. For background material on exponential dichotomy for differential equations, the books [8-10] may be consulted.

We note that the study of robustness in the case of exponential behavior has a long history. For the early work we can refer to Perron [1] and Massera and Schäffer [11]. For more recent works we refer to [12-15]. In particular, in [12], Barreira and Valls studied the existence of stable invariant manifold for any sufficiently small nonlinear perturbation of the linear equation. In [13], the authors discussed the parameter dependence of stable manifolds under nonuniform exponential dichotomy.

Recently, general stable and unstable behaviors with growth rates given by two increasing functions are exhibited by Bento and Silva [16]. The results in this work rely on the notion of nonuniform $(\mu, \nu)$-dichotomy, which includes the traditional exponential dichotomy and the polynomial dichotomy [17-19] and the $\rho$-nonuniform exponential dichotomy [20-22]. Since the nonuniform $(\mu, \nu)$-dichotomy is more general than nonuniform dichotomy before, so it is an emerging field drawing attention from both theoretical and applied disciplines. For example, in [23], the authors showed the robustness of nonuniform $(\mu, \nu)$-dichotomy provided that $\mu$ and $\nu$ were two differentiable functions. Authors of [24] showed the existence of invariant manifolds for sufficiently small Lipschitz perturbations of a linear equation with nonuniform $(\mu, \nu)$-dichotomy.

In the implementation of differential equations, time delay is a common phenomenon due to instantaneous perturbations. We can refer to the book [25] for functional differential equations. Recently, Barreira et al. [26] investigated the parameter dependence of stable manifolds for delay equations with polynomial dichotomies. It is assumed that $f$ is a sufficiently Lipschitz perturbation.

Our main aim is to show that the general behavior exhibited by a linear nonuniform $(\mu, \nu)$-dichotomy persists under nonlinear perturbed equations with parameter $\lambda$, where $\mu, v$ are two increasing functions. More precisely, we establish the existence of Lipschitz stable invariant for nonlinear perturbed equations with parameter $\lambda$ provided that the linear part has a nonuniform $(\mu, v)$-dichotomy and the nonlinear perturbations are sufficiently small. Our 
method is inspired in the former work in [26]. Meanwhile, an example is given to illustrate the applicability of the results.

The organization of this paper is as follows. In Section 2, we introduce delay linear differential equations and the perturbed equation with parameter $\lambda$. In Section 3, we consider the case of nonuniform $(\mu, \nu)$-contraction. In Section 4 , we show that the asymptotic stability of a nonuniform $(\mu, \nu)$ dichotomy persists under sufficiently small nonlinear perturbations with parameter $\lambda$. Finally, an example is provided to illustrate our theorems in Section 5.

\section{Preliminaries}

Given $r>0$, let $\chi=C\left([-r, 0], R^{n}\right)$ be the Banach space of continuous functions $\varphi:[-r, 0] \rightarrow R^{n}$ endowed with the norm

$$
\|\varphi\|=\sup _{\theta \in[-r, 0]}|\varphi(\theta)|,
$$

where $|\cdot|$ is the norm in $R^{n}$. Let $\bar{\chi}$ be the set of functions $\varphi:[-r, 0] \rightarrow R^{n}$ such that for each $s \in[-r, 0]$ the limits $\lim _{\theta \rightarrow s^{-}} \varphi(\theta)$ and $\lim _{\theta \rightarrow s^{+}} \varphi(\theta)$ exist, and $\lim _{\theta \rightarrow s^{+}} \varphi(\theta)=$ $\varphi(s)$. By [26], we see that $\bar{\chi}$ is a Banach space with the norm.

Given $s \geq 0$ and $\varphi \in \chi$, we consider the initial value problem

$$
x^{\prime}=L(t) x_{t}, \quad x_{s}=\varphi, \quad t \geq s,
$$

where $x_{t}(\theta)=x(t+\theta)$ for $\theta \in[-r, 0], L(t): \chi \rightarrow R^{n}$ are linear operators, and there is a $\vartheta>0$ such that

$$
\int_{t}^{t+r}\|L(\tau)\| d \tau \leq \vartheta(1+t),
$$

for all $t \geq 0$. For each $(s, \varphi) \in R^{+} \times \chi$, there is a unique solution $t \rightarrow x_{t}(\cdot, s, \varphi)$ of the initial value problem (2) with $x_{s}(\cdot, s, \varphi)=\varphi$. Define the evolution operator $T(t, s): \chi \rightarrow \chi$ associated with (2) by

$$
T(t, s) \varphi=x_{t}(\cdot, s, \varphi), \quad t \geq s .
$$

If $L(t)$ is written in the form

$$
L(t) \varphi=\int_{-r}^{0} d_{\theta}[\eta(t, \theta)] \varphi(\theta),
$$

where $\eta(t, \theta)$ is $n \times n$ matrices and is measurable in $(t, \theta) \epsilon$ $R^{+} \times[-r, 0]$ and continuous from the left in $\theta$, by [26], each linear operator $L(t)$ can be extended to $\bar{\chi}$ with the help of the integral in (5) in case that the Riemann-Stieltjes sums take value $[\eta(t, b)-\eta(t, a)] \varphi\left(b^{-}\right)$for each subinterval $t \rightarrow[a, b] \subset$ $[-r, 0]$. Thus for each $(s, \varphi) \in R^{+} \times \bar{\chi}$, there is a unique solution $t \rightarrow x_{t}(\cdot, s, \varphi) \subset \bar{\chi}$ of the integral equation obtained from (2) with $x_{s}(\cdot, s, \varphi)=\varphi$ and the corresponding evolution operator $\bar{T}(t, s): \bar{\chi} \rightarrow \bar{\chi}$ is defined by

$$
\bar{T}(t, s) \varphi=x_{t}(\cdot, s, \varphi), \quad t \geq s .
$$

It is easy to see that $\bar{T}(t, s) \mid \chi=T(t, s)$ and $\bar{T}(t, s) \bar{\chi} \subset \chi$ for $t \geq s+r$. We also consider the perturbed equation

$$
x^{\prime}=L(t) x_{t}+f\left(t, x_{t}, \lambda\right), \quad x_{s}=\varphi, \quad t \geq s,
$$

where $f: R^{+} \times \bar{\chi} \times Y \rightarrow R^{n}$ and $Y$ is an open subset of a Banach space (the parameter space).

\section{Stability for Nonuniform $(\mu, \nu)$-Contraction}

In this section, we show that the asymptotic stability of a nonuniform $(\mu, \nu)$ contraction persists under sufficiently small nonlinear perturbations. We say that an increasing function $u:[0, \infty) \rightarrow[1, \infty)$ is said to be a growth rate if $u(0)=1$ and $\lim _{t \rightarrow \infty} u(t)=\infty$. Let $\mu(t), v(t)$ be growth rates. Then we recall the definition of nonuniform $(\mu, \nu)$ contraction for (2).

Definition 1. Equation (2) is said to admit a nonuniform $(\mu, \nu)$-contraction if there exist constants $a<0, \varepsilon \geq 0$, and $K>1$ such that

$$
\|\bar{T}(t, s)\| \leq K\left[\frac{\mu(t)}{\mu(s)}\right]^{a} \nu(s)^{\varepsilon},
$$

for $t \geq s \geq 0$.

We assume that the following conditions hold:

$\left(H_{1}\right) f(t, 0, \lambda)=0$ for all $t \geq 0$ and $\lambda \in Y$;

$\left(H_{2}\right)$ there exist constants $c>0, \gamma>0$ such that for $t \geq 0$, $\varphi, \psi \in \bar{\chi}, \lambda, \eta \in Y$

$$
|f(t, \varphi, \lambda)-f(t, \psi, \lambda)| \leq c \nu(t)^{-\gamma}\|x-y\|,
$$$$
|f(t, \varphi, \lambda)-f(t, \varphi, \eta)| \leq c \nu(t)^{-\gamma}|\lambda-\eta| \cdot\|\varphi\| ;
$$

$\left(H_{3}\right)$ there exists $M \geq 0$ such that $M=\int_{0}^{\infty} \nu(\tau)^{\varepsilon-\gamma} d \tau<\infty$; $\left(H_{4}\right) c K M \leq 1 / 2$.

Theorem 2. Assume that (2) admits a nonuniform $(\mu, \nu)$ contraction. If $\left(H_{1}\right)-\left(H_{4}\right)$ hold, then for each $s \geq 0, \varphi \in \bar{\chi}$, the solutions $x_{t}^{\lambda}$ of (7) with initial condition $x_{s}^{\lambda}=\varphi$ satisfy

$$
\begin{gathered}
\left\|x_{t}^{\lambda}\right\| \leq \frac{K}{1-c K M}\left[\frac{\mu(t)}{\mu(s)}\right]^{a} \nu(s)^{\varepsilon}\|\varphi\|, \\
\left\|x_{t}^{\lambda}-x_{t}^{\mu}\right\| \leq \frac{c K^{2} M}{(1-c K M)^{2}}\left[\frac{\mu(t)}{\mu(s)}\right]^{a} \nu(s)^{\varepsilon}|\lambda-\mu| \cdot\|\varphi\|,
\end{gathered}
$$

for every $t \geq s$ and $\lambda, \mu \in Y$.

Proof. We see that the solution of (7) satisfies the following variation-of-parameters formula:

$$
x_{t}=\bar{T}(t, s) \varphi+\int_{s}^{t} \bar{T}(t, \tau) S_{0} f\left(\tau, x_{\tau}, \lambda\right) d \tau,
$$

where

$$
S_{0}(\theta)= \begin{cases}0, & -r \leq \theta<0 \\ I d, & \theta=0\end{cases}
$$

We consider the space $\mathscr{C}$ of function $x=x^{\lambda}:[s-r,+\infty) \rightarrow$ $R^{n}$ such that $x_{s}=\varphi, x_{t} \in \bar{\chi}$ for each $t \geq s$ and $\|x\|^{\prime} \leq 2 K$, where

$$
\|x\|^{\prime}=\sup \left\{\frac{\left\|x_{t}\right\|}{[\mu(t) / \mu(s)]^{a} \nu(s)^{\varepsilon}\|\varphi\|}: t \geq s\right\} .
$$


It is easy to see that $\mathscr{C}$ is a complete metric space. Set

$$
\begin{aligned}
& \left(J^{\lambda} x\right)(t+\theta) \\
& \quad=[\bar{T}(t, s) \varphi](\theta)+\int_{s}^{t} \bar{T}(t, \tau)\left(S_{0} f\left(\tau, x_{\tau}, \lambda\right)\right)(\theta) d \tau,
\end{aligned}
$$

for each $\lambda \in Y, x \in \mathscr{C}$, and $t \geq s$. Clearly, $J^{\lambda} x$ is continuous and $\left(J^{\lambda} x\right)_{s}=\varphi$ and $\left(J^{\lambda} x\right)_{t} \in \bar{\chi}$ for each $t \geq s$. By $\left(H_{1}\right)$, for each $x, y \in \mathscr{C}$, we have

$$
\begin{aligned}
& \left\|\left(J^{\lambda} x\right)_{t}-\left(J^{\lambda} y\right)_{t}\right\| \\
& \leq \int_{s}^{t}\|\bar{T}(t, \tau)\| \cdot\left|f\left(\tau, x_{\tau}, \lambda\right)-f\left(\tau, y_{\tau}, \lambda\right)\right| d \tau \\
& \leq c K\left[\frac{\mu(t)}{\mu(s)}\right]^{a} \nu(s)^{\varepsilon}\|\varphi\| \cdot\|x-y\|^{\prime} \int_{s}^{t} \nu(\tau)^{\varepsilon-\gamma} d \tau \\
& \quad \leq c K M\left[\frac{\mu(t)}{\mu(s)}\right]^{a} \nu(s)^{\varepsilon}\|\varphi\| \cdot\|x-y\|^{\prime} .
\end{aligned}
$$

Thus we have

$$
\left\|\left(J^{\lambda} x\right)_{t}-\left(J^{\lambda} y\right)_{t}\right\|^{\prime} \leq c K M\|x-y\|^{\prime} \leq \frac{1}{2}\|x-y\|^{\prime} .
$$

Then the operator $J^{\lambda}$ becomes a contraction. Furthermore, by $\left(H_{1}\right),\left(H_{2}\right)$, and $(8)$, we get

$$
\begin{aligned}
\left\|\left(J^{\lambda} x\right)_{t}\right\| \leq & \|\bar{T}(t, s) \varphi\|+\int_{s}^{t}\|\bar{T}(t, \tau)\| \cdot\left|f\left(\tau, x_{\tau}, \lambda\right)\right| d \tau \\
\leq & K\left[\frac{\mu(t)}{\nu(t)}\right]^{a} \nu(s)^{\varepsilon}\|\varphi\| \\
& +c K M\left[\frac{\mu(t)}{\mu(s)}\right]^{a} \nu(s)^{\varepsilon}\|\varphi\| \cdot\|x\|^{\prime} .
\end{aligned}
$$

Thus

$$
\left\|J^{\lambda} x\right\|^{\prime} \leq K+c K M\|x\|^{\prime} \leq K+\frac{1}{2}\|x\|^{\prime} \leq 2 K,
$$

which implies that $J^{\lambda}(\mathscr{C}) \subset \mathscr{C}$. Therefore, there exists a unique function $x \in \mathscr{C}$ such that $J^{\lambda} x=x$. By (18), we have

$$
\left\|x_{t}^{\lambda}\right\| \leq \frac{K}{1-c K M}\left[\frac{\mu(t)}{\mu(s)}\right]^{a} \nu(s)^{\varepsilon}\|\varphi\|,
$$

for each $t \geq s$. By (19) and $\left(H_{2}\right)$, we get

$$
\begin{aligned}
\left|f\left(\tau, x_{\tau}^{\lambda}, \lambda\right)-f\left(\tau, x_{\tau}^{\eta}, \eta\right)\right| \\
=\left|f\left(\tau, x_{\tau}^{\lambda}, \lambda\right)-f\left(\tau, x_{\tau}^{\lambda}, \eta\right)\right| \\
\quad+\left|f\left(\tau, x_{\tau}^{\lambda}, \eta\right)-f\left(\tau, x_{\tau}^{\eta}, \eta\right)\right| \\
\leq c \nu(\tau)^{-\gamma}\left\|x_{\tau}^{\eta}\right\| \cdot|\lambda-\eta|+c \nu(\tau)^{-\gamma}\left\|x_{\tau}^{\lambda}-x_{\tau}^{\eta}\right\| \\
\leq \frac{c K}{1-c K M}\left[\frac{\mu(\tau)}{\mu(s)}\right]^{a} \nu(s)^{\varepsilon} \nu(\tau)^{-\gamma}\|\varphi\| \cdot|\lambda-\eta| \\
\quad+c\left[\frac{\mu(\tau)}{\mu(s)}\right]^{a} \nu(s)^{\varepsilon} \nu(\tau)^{-\gamma}\|\varphi\| \cdot\left\|x^{\lambda}-x^{\eta}\right\|^{\prime} .
\end{aligned}
$$

Therefore, it follows that

$$
\begin{aligned}
\left\|x_{t}^{\lambda}-x_{t}^{\mu}\right\| & \\
\leq & \int_{s}^{t}\|\bar{T}(t, \tau)\| \cdot\left|f\left(\tau, x_{\tau}^{\lambda}, \lambda\right)-f\left(\tau, x_{\tau}^{\mu}, \mu\right)\right| d \tau \\
\leq & \frac{c K^{2}}{1-c K M}\left[\frac{\mu(t)}{\mu(s)}\right]^{a} \nu(s)^{\varepsilon}\|\varphi\| \cdot|\lambda-\eta| \int_{s}^{t} \nu(\tau)^{\varepsilon-\gamma} d \tau \\
& +c K\left[\frac{\mu(t)}{\mu(s)}\right]^{a} \nu(s)^{\varepsilon}\|\varphi\| \cdot\left\|x^{\lambda}-x^{\eta}\right\|^{\prime} \int_{s}^{t} \nu(\tau)^{\varepsilon-\gamma} d \tau \\
\leq & \frac{c K^{2} M}{1-c K M}\left[\frac{\mu(t)}{\mu(s)}\right]^{a} \nu(s)^{\varepsilon}\|\varphi\| \cdot|\lambda-\eta| \\
& +c K M\left[\frac{\mu(t)}{\mu(s)}\right]^{a} \nu(s)^{\varepsilon}\|\varphi\| \cdot\left\|x^{\lambda}-x^{\eta}\right\|^{\prime}
\end{aligned}
$$

which yields that

$$
\left\|x^{\lambda}-x^{\mu}\right\|^{\prime} \leq \frac{c K^{2} M}{1-c K M}|\lambda-\eta|+c K M\left\|x^{\lambda}-x^{\eta}\right\|^{\prime} .
$$

This completes the proof of the theorem.

\section{Stable Invariant Manifolds}

In this section, we establish stable manifolds theorem under sufficiently small perturbation of nonuniform $(\mu, \nu)$ dichotomy.

Definition 3. Equation (2) is said to admit a nonuniform $(\mu, \nu)$-dichotomy if there exist projections $P(t): \bar{\chi} \rightarrow \bar{\chi}$ and constants $a<0 \leq b, \varepsilon \geq 0$, and $K>1$ such that for each $t \geq s \geq 0$

(1) $P(t) \bar{T}(t, s)=\bar{T}(t, s) P(s)$;
(2) letting $Q(t)=I d-P(t)$, the map

$$
\bar{T}_{\mathrm{Q}}(t, s):=\bar{T}(t, s) \mid Q(s) \bar{\chi}: Q(s) \bar{\chi} \longrightarrow Q(t) \bar{\chi}
$$

is invertible;

(3)

$$
\begin{aligned}
& \|\bar{T}(t, s) P(s)\| \leq K\left[\frac{\mu(t)}{\mu(s)}\right]^{a} \nu(s)^{\varepsilon}, \\
& \left\|\bar{T}_{Q}(t, s)^{-1} Q(t)\right\| \leq K\left[\frac{\mu(t)}{\mu(s)}\right]^{-b} \nu(t)^{\varepsilon} .
\end{aligned}
$$

Setting the linear subspace

$$
E(t)=P(t)(\bar{\chi}), \quad F(t)=Q(t)(\bar{\chi}) .
$$

Let $\mathscr{B}$ be the space of continuous functions $\Phi: E \rightarrow \bar{\chi}$ such that, for each $s \geq 0, \Phi(s, 0)=0, \Phi(s, E(s)) \subset F(s)$, and

$$
\|\Phi(s, \varphi)-\Phi(s, \psi)\| \leq\|\varphi-\psi\|, \quad \varphi, \psi \in E(s),
$$


with the norm

$$
\|\Phi\|=\sup \left\{\frac{\|\Phi(s, \varphi)\|}{\|\varphi\|}: s \geq 0, \varphi \in E(s) \backslash\{0\}\right\} .
$$

Moreover, we denote by $\mathscr{B}$ the space of continuous functions $\widehat{\Phi}: Y \rightarrow E(s)$, which can be written by $\widehat{\Phi}(\lambda)=\Phi^{\lambda}$, such that

$$
\left\|\Phi^{\lambda}(s, \varphi)-\Phi^{\eta}(s, \varphi)\right\| \leq|\lambda-\eta| \cdot\|\varphi\|,
$$

for each $s \geq 0, \varphi \in E(s)$, and $\lambda, \eta \in Y$. Given $\widehat{\Phi} \in \mathscr{B}$ and $\lambda \in Y$, we consider the set

$$
\mathscr{W}^{\lambda}=\left\{s, \varphi, \Phi^{\lambda}(s, \varphi): s \geq 0, \varphi \in E(s)\right\} .
$$

Assume that (2) admits a nonuniform $(\mu, \nu)$-dichotomy, for each $\lambda \in Y$, the solution $\left(x_{t}, y_{t}\right) \in E(t) \times F(t)$ of (7) with initial condition $\left(x_{s}, x_{s}\right) \in E(s) \times F(s)$ satisfies

$$
\begin{aligned}
& x_{t}=\bar{T}(t, s) x_{s}+\int_{s}^{t} \bar{T}(t, \tau) P(\tau) S_{0} f\left(\tau, x_{\tau}, y_{\tau}, \lambda\right) d \tau, \\
& y_{t}=\bar{T}(t, s) y_{s}+\int_{s}^{t} \bar{T}(t, \tau) Q(\tau) S_{0} f\left(\tau, x_{\tau}, y_{\tau}, \lambda\right) d \tau .
\end{aligned}
$$

We consider the semiflow

$$
\Omega_{\epsilon}^{\lambda}\left(s, x_{s}, y_{s}\right)=\left(s+\epsilon, x_{s+\epsilon}, y_{s+\epsilon}\right),
$$

which is generated by the following equation:

$$
t^{\prime}=1, \quad x^{\prime}=L(t) x_{t}+f\left(t, x_{t}, \lambda\right) .
$$

To obtain our results, we also need the following assumptions:

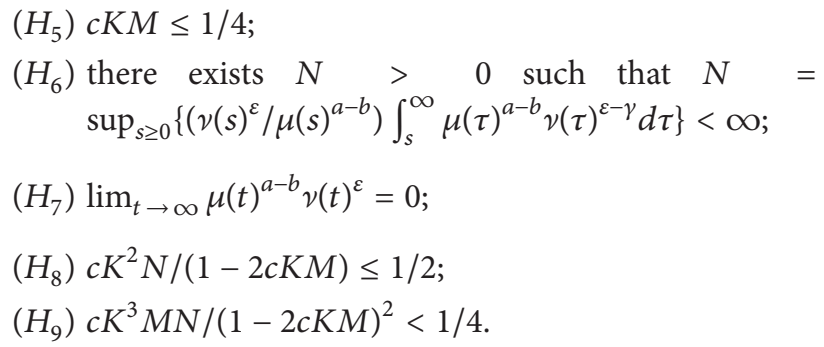

Theorem 4. Assume that (2) admits a nonuniform $(\mu, \nu)$ dichotomy. If $\left(\mathrm{H}_{1}\right)-\left(\mathrm{H}_{3}\right)$ and $\left(\mathrm{H}_{5}\right)-\left(\mathrm{H}_{9}\right)$ hold, then for each $\delta>$ 0 sufficiently small, there exists a unique function $\widehat{\Phi}=\Phi^{\lambda} \in \mathscr{B}$ such that

$$
\Omega_{\epsilon}^{\lambda}\left(s, \varphi, \Phi^{\lambda}(s, \varphi)\right) \in \mathscr{W}^{\lambda}, \quad \text { for each } \epsilon \geq 0, \lambda \in Y .
$$

Furthermore, for each $\epsilon=t-s \geq 0, \varphi, \psi \in E(s)$, and $\lambda, \eta \in Y$

$$
\begin{aligned}
\left\|\Omega_{\epsilon}^{\lambda}\left(s, \varphi, \Phi^{\lambda}(s, \varphi)\right)-\Omega_{\epsilon}^{\lambda}\left(s, \psi, \Phi^{\lambda}(s, \psi)\right)\right\| \\
\leq \frac{2 K}{1-2 c K M}\left[\frac{\mu(t)}{\mu(s)}\right]^{a} \nu(s)^{\varepsilon}\|\varphi-\psi\|, \\
\left\|\Omega_{\epsilon}^{\lambda}\left(s, \varphi, \Phi^{\lambda}(s, \varphi)\right)-\Omega_{\epsilon}^{\eta}\left(s, \varphi, \Phi^{\eta}(s, \varphi)\right)\right\| \\
\leq\left[\frac{6 c K^{2} M}{(1-2 c K M)^{2}}+\frac{K}{1-2 c K M}\right]\left[\frac{\mu(t)}{\mu(s)}\right]^{a} \nu(s)^{\varepsilon}\|\varphi\| \\
\quad \cdot|\lambda-\eta| .
\end{aligned}
$$

Proof. We replace (30) by

$$
\begin{aligned}
x_{t}=\bar{T}(t, s) \varphi & +\int_{s}^{t} \bar{T}(t, \tau) P(\tau) S_{0} f\left(\tau, x_{\tau}, \Phi^{\lambda}\left(\tau, x_{\tau}\right), \lambda\right) d \tau \\
\Phi^{\lambda}\left(t, x_{t}\right)= & \bar{T}(t, s) \Phi^{\lambda}(s, \varphi) \\
& +\int_{s}^{t} \bar{T}(t, \tau) Q(\tau) S_{0} f\left(\tau, x_{\tau}, \Phi^{\lambda}\left(\tau, x_{\tau}\right), \lambda\right) d \tau
\end{aligned}
$$

Lemma 5. For each $\lambda \in Y, \widehat{\Phi}=\Phi^{\lambda} \in \mathscr{B}$, given $(s, \varphi) \epsilon$ $R^{+} \times E(s)$, there is a unique function $x^{(\lambda, \widehat{\Phi}, \varphi)}:[s-r,+\infty) \rightarrow R^{n}$ with $x_{s}^{(\lambda, \widehat{\Phi}, \varphi)}=\varphi$ and $x_{t}^{(\lambda, \widehat{\Phi}, \varphi)} \in E(t)$, satisfying (36). Moreover, for $t \geq s$

$$
\left\|x_{t}^{(\lambda, \widehat{\Phi}, \varphi)}\right\| \leq \frac{K}{1-2 c K M}\left[\frac{\mu(t)}{\mu(s)}\right]^{a} \nu(s)^{\varepsilon}\|\varphi\| .
$$

Proof. We consider the operator $\bar{J}^{\lambda}$ defined by

$$
\begin{aligned}
\left(\bar{J}^{\lambda} x\right)_{t}= & \bar{T}(t, s) \varphi \\
& +\int_{s}^{t} \bar{T}(t, \tau) P(\tau) S_{0} f\left(\tau, x_{\tau}, \Phi^{\lambda}\left(\tau, x_{\tau}\right), \lambda\right) d \tau,
\end{aligned}
$$

in the space $\mathscr{A}$ of functions $x=x^{(\lambda, \widehat{\Phi}, \varphi)}:[s-r,+\infty) \rightarrow R^{n}$ such that $x_{s}^{(\lambda, \widehat{\Phi}, \varphi)}=\varphi, x_{t}^{(\lambda, \Phi, \varphi)} \in E(t)$ for each $t \geq s$ and $\|x\|^{\prime} \leq$ $2 K$, where $\|x\|^{\prime}$ is given by (13). We see that $\mathscr{A}$ is a complete metric space with the norm $\|x\|^{\prime}$. For each $x, y \in \mathscr{A}$ and $t \geq s$, by $\left(H_{2}\right)$ and $(26)$, we obtain

$$
\begin{aligned}
& \left|f\left(\tau, x_{\tau}, \Phi^{\lambda}\left(\tau, x_{\tau}\right), \lambda\right)-f\left(\tau, y_{\tau}, \Phi^{\lambda}\left(\tau, y_{\tau}\right), \lambda\right)\right| \\
& \quad \leq c \nu(\tau)^{-\gamma}\left\|\left(x_{\tau}-y_{\tau}, \Phi^{\lambda}\left(\tau, x_{\tau}\right)-\Phi^{\lambda}\left(\tau, y_{\tau}\right)\right)\right\| \\
& \quad \leq 2 c\left[\frac{\mu(\tau)}{\mu(s)}\right]^{a} \nu(s)^{\varepsilon} \nu(\tau)^{-\gamma}\|\varphi\| \cdot\|x-y\|^{\prime} .
\end{aligned}
$$

By (39), (40), and (24), we have

$$
\begin{aligned}
& \left\|\left(\bar{J}^{\lambda} x\right)_{t}-\left(\bar{J}^{\lambda} y\right)_{t}\right\| \\
& \leq \int_{s}^{t}\|\bar{T}(t, \tau) P(\tau)\| \cdot \mid f\left(\tau, x_{\tau}, \Phi^{\lambda}\left(\tau, x_{\tau}\right), \lambda\right) \\
& \quad-f\left(\tau, y_{\tau}, \Phi^{\lambda}\left(\tau, y_{\tau}\right), \lambda\right) \mid d \tau \\
& \leq 2 c K\left[\frac{\mu(t)}{\mu(s)}\right]^{a} \nu(s)^{\varepsilon}\|\varphi\| \cdot\|x-y\|^{\prime} \int_{s}^{t} \nu(\tau)^{\varepsilon-\gamma} d \tau \\
& \leq 2 c K M\left[\frac{\mu(t)}{\mu(s)}\right]^{a} \nu(s)^{\varepsilon}\|\varphi\| \cdot\|x-y\|^{\prime} .
\end{aligned}
$$


Therefore

$$
\left\|\bar{J}^{\lambda} x-\bar{J}^{\lambda} y\right\|^{\prime} \leq 2 c K M\|x-y\|^{\prime} .
$$

By $\left(\mathrm{H}_{2}\right)$ and (26), we obtain

$$
\begin{aligned}
\| f & \left(\tau, x_{\tau}, \Phi^{\lambda}\left(\tau, x_{\tau}\right), \lambda\right) \| \\
& \leq c \nu(\tau)^{-\gamma}\left\|\left(x_{\tau}, \Phi^{\lambda}\left(\tau, x_{\tau}\right)\right)\right\| \\
& \leq c \nu(\tau)^{-\gamma}\left(\left\|x_{\tau}\right\|+\left\|\Phi\left(\tau, x_{\tau}\right)\right\|\right) \leq 2 c \nu(\tau)^{-\gamma}\left\|x_{\tau}\right\| .
\end{aligned}
$$

Thus

$$
\begin{aligned}
\left\|\left(\bar{J}^{\lambda} x\right)_{t}\right\| \leq & \|\bar{T}(t, s) \varphi\| \\
& +\int_{s}^{t}\|\bar{T}(t, \tau) P(\tau)\| \cdot\left|f\left(\tau, x_{\tau}, \Phi^{\lambda}\left(\tau, x_{\tau}\right), \lambda\right)\right| d \tau \\
\leq & K\left[\frac{\mu(t)}{\mu(s)}\right]^{a} v(s)^{\varepsilon}\|\varphi\| \\
& +2 c K\left[\frac{\mu(t)}{\mu(s)}\right]^{a} v(s)^{\varepsilon}\|x\|^{\prime} \int_{s}^{t} v(\tau)^{\varepsilon-\gamma} d \tau \\
\leq & K\left[\frac{\mu(t)}{\mu(s)}\right]^{a} \nu(s)^{\varepsilon}\|\varphi\|+2 c K M\left[\frac{\mu(t)}{\mu(s)}\right]^{a} \nu(s)^{\varepsilon}\|x\|^{\prime} .
\end{aligned}
$$

The remainder of proof is proceeded the same as Theorem 2; here we omit it.

Lemma 6. Given $\widehat{\Phi}=\Phi^{\lambda} \in \mathscr{B}$, for each $\lambda \in Y, \varphi, \psi \in E(s)$, one has

$$
\left\|x_{t}^{(\lambda, \widehat{\Phi}, \varphi)}-x_{t}^{(\lambda, \widehat{\Phi}, \psi)}\right\| \leq \frac{K}{1-2 c k M}\left[\frac{\mu(t)}{\mu(s)}\right]^{a} \nu(s)^{\varepsilon}\|\varphi-\psi\| .
$$

Proof. By $\left(\mathrm{H}_{2}\right)$ and (26), for each $\tau \geq s$, we get

$$
\begin{aligned}
q_{1}(t):= & \mid f\left(\tau, x_{\tau}^{(\lambda, \widehat{\Phi}, \varphi)}, \Phi^{\lambda}\left(\tau, x_{\tau}^{(\lambda, \widehat{\Phi}, \varphi)}\right), \lambda\right) \\
& -f\left(\tau, x_{\tau}^{(\lambda, \widehat{\Phi}, \psi)}, \Phi^{\lambda}\left(\tau, x_{\tau}^{(\lambda, \widehat{\Phi}, \psi)}\right), \lambda\right) \mid \\
\leq & 2 c \nu(\tau)^{-\gamma}\left\|x_{\tau}^{(\lambda, \widehat{\Phi}, \varphi)}-x_{\tau}^{(\lambda, \widehat{\Phi}, \psi)}\right\| \\
\leq & 2 c\left[\frac{\mu(\tau)}{\mu(s)}\right]^{a} \nu(s)^{\varepsilon} \nu(\tau)^{-\gamma}\left\|x^{(\lambda, \widehat{\Phi}, \varphi)}-x^{(\lambda, \widehat{\Phi}, \psi)}\right\|^{\prime} .
\end{aligned}
$$

By (39), (46), and (24), we have

$$
\begin{aligned}
& \left\|x_{t}^{(\lambda, \widehat{\Phi}, \varphi)}-x_{t}^{(\lambda, \widehat{\Phi}, \psi)}\right\| \\
& \leq\|\bar{T}(t, s) P(s)\| \cdot\|\varphi-\psi\| \\
& \quad+\int_{s}^{t}\|\bar{T}(t, \tau) P(\tau)\| q_{1}(\tau) d \tau
\end{aligned}
$$

$$
\begin{aligned}
\leq & K\left[\frac{\mu(t)}{\mu(s)}\right]^{a} \nu(s)^{\varepsilon}\|\varphi-\psi\| \\
& +2 c K\left[\frac{\mu(t)}{\mu(s)}\right]^{a} v(s)^{\varepsilon} \\
& \times\left\|x^{(\lambda, \widehat{\Phi}, \varphi)}-x^{(\lambda, \widehat{\Phi}, \psi)}\right\|^{\prime} \int_{s}^{t} \nu(\tau)^{\varepsilon-\gamma} d \tau \\
\leq & K\left[\frac{\mu(t)}{\mu(s)}\right]^{a} \nu(s)^{\varepsilon}\|\varphi-\psi\| \\
& +2 c K M\left[\frac{\mu(t)}{\mu(s)}\right]^{a} \nu(s)^{\varepsilon}\left\|x^{(\lambda, \widehat{\Phi}, \varphi)}-x^{(\lambda, \widehat{\Phi}, \psi)}\right\|^{\prime},
\end{aligned}
$$

which implies that inequality (45) holds.

Lemma 7. Given $\varphi \in E(s)$, for each $\lambda \in Y, \widehat{\Phi}=\Phi^{\lambda}, \widehat{\Psi}=$ $\Psi^{\lambda}, \widehat{\Phi}, \widehat{\Psi} \in \mathscr{B}$, one has

$$
\begin{aligned}
& \left\|x_{t}^{(\lambda, \widehat{\Phi}, \varphi)}-x_{t}^{(\lambda, \widehat{\Psi}, \varphi)}\right\| \\
& \quad \leq \frac{c K^{2} M}{(1-2 c K M)^{2}}\left[\frac{\mu(t)}{\mu(s)}\right]^{a} \nu(s)^{\varepsilon}\|\varphi\| \cdot\|\widehat{\Phi}-\widehat{\Psi}\| .
\end{aligned}
$$

Proof. By $\left(H_{2}\right)$ and (26), for each $\tau \geq s$, we get

$$
\begin{aligned}
& q_{2}(\tau):=\mid f\left(\tau, x_{\tau}^{(\lambda, \widehat{\Phi}, \varphi)}, \Phi^{\lambda}\left(\tau, x_{\tau}^{(\lambda, \widehat{\Phi}, \varphi)}\right), \lambda\right) \\
& -f\left(\tau, x_{\tau}^{(\lambda, \widehat{,}, \varphi)}, \Psi^{\lambda}\left(\tau, x_{\tau}^{(\lambda, \widehat{\Psi}, \varphi)}\right), \lambda\right) \mid \\
& \leq c \nu(\tau)^{-\gamma} \|\left(x_{\tau}^{(\lambda, \widehat{\Phi}, \varphi)}-x_{\tau}^{(\lambda, \widehat{\Psi}, \varphi)}, \Phi^{\lambda}\left(\tau, x_{\tau}^{(\lambda, \widehat{\Phi}, \varphi)}\right)\right. \\
& \left.-\Psi^{\lambda}\left(\tau, x_{\tau}^{(\lambda, \widehat{\Psi}, \varphi)}\right)\right) \| \\
& \leq c \nu(\tau)^{-\gamma}\left[\left\|x_{\tau}^{(\lambda, \widehat{\Phi}, \varphi)}-x_{\tau}^{(\lambda, \widehat{\Psi}, \varphi)}\right\|\right. \\
& \left.+\left\|\Phi^{\lambda}\left(\tau, x_{\tau}^{(\lambda, \widehat{\Phi}, \varphi)}\right)-\Psi^{\lambda}\left(\tau, x_{\tau}^{(\lambda, \widehat{\Psi}, \varphi)}\right)\right\|\right], \\
& \left\|\Phi^{\lambda}\left(\tau, x_{\tau}^{(\lambda, \widehat{\Phi}, \varphi)}\right)-\Psi^{\lambda}\left(\tau, x_{\tau}^{(\lambda, \widehat{\Psi}, \varphi)}\right)\right\| \\
& \leq\left\|\Phi^{\lambda}\left(\tau, x_{\tau}^{(\lambda, \widehat{\Phi}, \varphi)}\right)-\Phi^{\lambda}\left(\tau, x_{\tau}^{(\lambda, \widehat{\Psi}, \varphi)}\right)\right\| \\
& +\left\|\Phi^{\lambda}\left(\tau, x_{\tau}^{(\lambda, \widehat{\Psi}, \varphi)}\right)-\Psi^{\lambda}\left(\tau, x_{\tau}^{(\lambda, \widehat{\Psi}, \varphi)}\right)\right\| \\
& \leq\left\|x_{\tau}^{(\lambda, \widehat{\Phi}, \varphi)}-x_{\tau}^{(\lambda, \widehat{\Psi}, \varphi)}\right\|+\left\|x_{\tau}^{(\lambda, \widehat{\Phi}, \varphi)}\right\| \cdot\|\widehat{\Phi}-\widehat{\Psi}\| \\
& \leq\left[\frac{\mu(\tau)}{\mu(s)}\right]^{a} v(s)^{\varepsilon}\|\varphi\| \cdot\left\|x^{(\lambda, \widehat{\Phi}, \varphi)}-x^{(\lambda, \widehat{\Psi}, \varphi)}\right\|^{\prime} \\
& +\left\|x_{\tau}^{(\lambda, \widehat{\Phi}, \varphi)}\right\| \cdot\|\widehat{\Phi}-\widehat{\Psi}\| .
\end{aligned}
$$


Then, by (24), (49), and Lemma 5, we obtain

$$
\begin{aligned}
& \left\|x_{t}^{(\lambda, \widehat{\Phi}, \varphi)}-x_{t}^{(\lambda, \widehat{\Psi}, \varphi)}\right\| \\
& \leq \int_{s}^{t}\|\bar{T}(t, \tau) P(\tau)\| q_{2}(\tau) d \tau \\
& \leq 2 c K\left[\frac{\mu(t)}{\mu(s)}\right]^{a} \nu(s)^{\varepsilon}\|\varphi\| \\
& \quad \| x^{(\lambda, \widehat{\Phi}, \varphi)}-x^{(\lambda, \widehat{\Psi}, \varphi) \|^{\prime} \int_{s}^{t} \nu(\tau)^{\varepsilon-\gamma} d \tau} \\
& +\frac{c K^{2}}{1-2 c K M}\left[\frac{\mu(t)}{\mu(s)}\right]^{a} \nu(s)^{\varepsilon}\|\varphi\| \\
& \quad \cdot\|\widehat{\Phi}-\widehat{\Psi}\| \int_{s}^{t} \nu(\tau)^{\varepsilon-\gamma} d \tau \\
& \leq 2 c K M\left[\frac{\mu(t)}{\mu(s)}\right]^{a} \nu(s)^{\varepsilon}\|\varphi\| \cdot\left\|x^{(\lambda, \widehat{\Phi}, \varphi)}-x^{(\lambda, \widehat{\Psi}, \varphi)}\right\|^{\prime} \\
& +\frac{c K^{2} M}{1-2 c K M}\left[\frac{\mu(t)}{\mu(s)}\right]^{a} v(s)^{\varepsilon}\|\varphi\| \cdot\|\widehat{\Phi}-\widehat{\Psi}\| \cdot
\end{aligned}
$$

Therefore, inequality (48) holds for $t \geq s \geq 0$.

Next, we transform (37) into an equivalent problem.

Lemma 8. Given $\widehat{\Phi}=\Phi^{\lambda} \in \mathscr{B}$, the following properties hold.

(1) If for each $s \geq 0, \varphi \in E(s), t \geq s$

$$
\begin{aligned}
\Phi^{\lambda} & \left(t, x_{t}^{(\lambda, \widehat{\Phi}, \varphi)}\right) \\
= & \bar{T}(t, s) \widehat{\Phi}(s, \varphi) \\
& +\int_{s}^{t} \bar{T}(t, \tau) Q(\tau) S_{0} f\left(\tau, x_{\tau}^{(\lambda, \widehat{\Phi}, \varphi)}, \Phi^{\lambda}\left(\tau, x_{\tau}^{(\lambda, \widehat{\Phi}, \varphi)}\right), \lambda\right) d \tau,
\end{aligned}
$$

then

$$
\begin{aligned}
\Phi^{\lambda}(s, \varphi)=-\int_{s}^{\infty} \bar{T}(\tau, s)^{-1} Q(\tau) S_{0} f\left(\tau, x_{\tau}^{(\lambda, \widehat{\Phi}, \varphi)},\right. \\
\left.\Phi^{\lambda}\left(\tau, x_{\tau}^{(\lambda, \widehat{\Phi}, \varphi)}\right), \lambda\right) d \tau .
\end{aligned}
$$

(2) If identity (52) holds for each $s \geq 0, \varphi \in E(s)$, then (51) holds for each $s \geq 0, \varphi \in E(s)$, and $t \geq s$.

Proof. By $\left(H_{1}\right),\left(H_{2}\right),(26)$, and Lemma 5, we have

$$
\begin{aligned}
& \left|f\left(\tau, x_{\tau}^{(\lambda, \widehat{\Phi}, \varphi)}, \Phi^{\lambda}\left(\tau, x_{\tau}^{(\lambda, \widehat{\Phi}, \varphi)}\right), \lambda\right)\right| \\
& \quad \leq 2 c \nu(\tau)^{-\gamma}\left\|x_{\tau}^{(\lambda, \widehat{\Phi}, \varphi)}\right\| \\
& \quad \leq \frac{2 c K}{1-2 c K M}\left[\frac{\mu(\tau)}{\mu(s)}\right]^{a} \nu(s)^{\varepsilon} \nu(\tau)^{-\gamma}\|\varphi\| .
\end{aligned}
$$

Hence

$$
\begin{aligned}
\int_{s}^{\infty} & \left\|T(\tau, s)^{-1} Q(\tau)\right\| \\
& \cdot\left|f\left(\tau, x_{\tau}^{(\lambda, \widehat{\Phi}, \varphi)}, \Phi^{\lambda}\left(\tau, x_{\tau}^{(\lambda, \widehat{\Phi}, \varphi)}\right), \lambda\right)\right| d \tau \\
\leq & 2 c K\|\varphi\| \int_{s}^{\infty}\left(\frac{\mu(\tau)}{\mu(s)}\right)^{a-b} \nu(\tau)^{\varepsilon-\gamma} \nu(s)^{\varepsilon} d \tau \\
\leq & 2 c K N\|\varphi\|<\infty .
\end{aligned}
$$

Therefore, the right-hand of (52) is well defined. We assume that (51) holds for each $s \geq 0, \varphi \in E(s)$, and $t \geq s$; then it follows that

$$
\begin{gathered}
\Phi^{\lambda}(s, \varphi)=\bar{T}_{\mathrm{Q}}(t, s)^{-1} \mathrm{Q}(t) \Phi^{\lambda}\left(t, x_{t}^{(\lambda, \widehat{\Phi}, \varphi)}\right) \\
-\int_{s}^{t} \bar{T}_{\mathrm{Q}}(\tau, s)^{-1} \mathrm{Q}(\tau) S_{0} f\left(\tau, x_{\tau}^{(\lambda, \widehat{\Phi}, \varphi)},\right. \\
\left.\Phi^{\lambda}\left(\tau, x_{\tau}^{(\lambda, \widehat{\Phi}, \varphi)}\right), \lambda\right) d \tau .
\end{gathered}
$$

By (24), (26), and Lemma 5, we have

$$
\begin{aligned}
& \left\|\bar{T}_{Q}(t, s)^{-1} Q(t) \Phi^{\lambda}\left(t, x_{t}^{(\lambda, \widehat{\Phi}, \varphi)}\right)\right\| \\
& \quad \leq \frac{K^{2}}{1-2 c K M}\left[\frac{\mu(t)}{\mu(s)}\right]^{a-b} \nu(t)^{\varepsilon} \nu(s)^{\varepsilon}\|\varphi\| .
\end{aligned}
$$

Letting $t \rightarrow \infty$ in (55), it follows from $\left(H_{7}\right)$ that for each $s \geq 0, \varphi \in E(s)$, and $t \geq s$, (52) holds.

If (52) holds for every $s \geq 0, \varphi \in E(s)$, and $t \geq s$, we have

$$
\begin{gathered}
\bar{T}(t, s) \Phi^{\lambda}(s, \varphi) \\
+\int_{s}^{t} \bar{T}_{\mathrm{Q}}(t, \tau) Q(\tau) S_{0} f\left(\tau, x_{\tau}^{(\lambda, \widehat{\Phi}, \varphi)},\right. \\
\left.\Phi^{\lambda}\left(\tau, x_{\tau}^{(\lambda, \widehat{\Phi}, \varphi)}\right), \lambda\right) d \tau \\
=-\int_{t}^{\infty} \bar{T}_{\mathrm{Q}}(\tau, t)^{-1} \mathrm{Q}(\tau) S_{0} f\left(\tau, x_{\tau}^{(\lambda, \widehat{\Phi}, \varphi)},\right. \\
\left.\Phi^{\lambda}\left(\tau, x_{\tau}^{(\lambda, \widehat{\Phi}, \varphi)}\right), \lambda\right) d \tau .
\end{gathered}
$$

Replacing $(s, \varphi)$ by $\left(t, x_{t}^{(\lambda, \widehat{\Phi}, \varphi)}\right)$ in $(52)$, we have

$$
\begin{gathered}
\Phi^{\lambda}\left(t, x_{t}^{(\lambda, \widehat{\Phi}, \varphi)}\right) \\
=-\int_{t}^{\infty} \bar{T}_{Q}(\tau, t)^{-1} Q(\tau) S_{0} f\left(\tau, x_{\tau}^{(\lambda, \widehat{\Phi}, \varphi)},\right. \\
\left.\Phi^{\lambda}\left(\tau, x_{\tau}^{(\lambda, \widehat{\Phi}, \varphi)}\right), \lambda\right) d \tau .
\end{gathered}
$$

From (57) and (58), we see that (51) holds for $(s, \varphi) \in R^{+} \times$ $E(s)$. 
Lemma 9. For each $\lambda \in Y$, there exists a unique function $\widehat{\Phi}=$ $\Phi^{\lambda} \in \mathscr{B}$ such that (52) holds for every $s \geq 0, \varphi \in E(s)$.

Proof. We consider the operator $\hat{J}^{\lambda}$ defined for each $\Phi \in \mathscr{B}$ by

$$
\begin{aligned}
& \left(\widehat{J}^{\lambda} \Phi^{\lambda}\right)(s, \varphi) \\
& =-\int_{s}^{\infty} T_{Q}(\tau, s)^{-1} Q(\tau) S_{0} f\left(\tau, x_{\tau}^{(\lambda, \widehat{\Phi}, \varphi)},\right. \\
& \left.\Phi^{\lambda}\left(\tau, x_{\tau}^{(\lambda, \widehat{\Phi}, \varphi)}\right), \lambda\right) d \tau,
\end{aligned}
$$

for every $s \geq 0, \varphi \in E(s)$. When $\varphi=0$, we have $x_{\tau}^{(\lambda, \widehat{\Phi}, \varphi)}=0$; then $\left(\widehat{J}^{\lambda} \Phi^{\lambda}\right)(s, 0)=0$. By (24), (46), and Lemma 6, we have

$$
\begin{aligned}
& \left\|\left(\widehat{J}^{\lambda} \Phi^{\lambda}\right)(s, \varphi)-\left(\widehat{J}^{\lambda} \Phi^{\lambda}\right)(s, \psi)\right\| \\
& \leq \int_{s}^{\infty}\left\|\bar{T}(\tau, s)^{-1} Q(\tau)\right\| \\
& \cdot \mid f\left(\tau, x_{\tau}^{(\lambda, \widehat{\Phi}, \varphi)}, \Phi^{\lambda}\left(\tau, x_{\tau}^{(\lambda, \widehat{\Phi}, \varphi)}\right), \lambda\right) \\
& \quad-f\left(\tau, x_{\tau}^{(\lambda, \widehat{\Phi}, \psi)}, \Phi^{\lambda}\left(\tau, x_{\tau}^{(\lambda, \widehat{\Phi}, \psi)}\right), \lambda\right) \mid d \tau \\
& \leq \frac{2 c K^{2}}{1-2 c K M}\|\varphi-\psi\| \\
& \quad \times \int_{s}^{\infty}\left[\frac{\mu(\tau)}{\mu(s)}\right]^{a-b} \nu(s)^{\varepsilon} \nu(\tau)^{\varepsilon-\gamma} d \tau \\
& \leq \frac{2 c K^{2} N}{1-2 c K M}\|\varphi-\psi\| .
\end{aligned}
$$

By $\left(H_{8}\right)$, for each $s \geq 0, \varphi, \psi \in E(s)$, we can conclude that

$$
\left\|\left(J^{\lambda} \Phi^{\lambda}\right)(s, \varphi)-\left(J^{\lambda} \Phi^{\lambda}\right)(s, \psi)\right\| \leq\|\varphi-\psi\|,
$$

which implies that $\widehat{J}^{\lambda}(\mathscr{B}) \subset \mathscr{B}$.

The next step is done that $\widehat{J}^{\lambda}$ is a contraction. Given $\varphi \in$ $E(s)$, for each $\widehat{\Phi}=\Phi^{\lambda}, \widehat{\Psi}=\Psi^{\lambda}$, and $\Phi^{\lambda}, \Psi^{\lambda} \in \mathscr{B}$, by (49), Lemmas 5 , and 7 , we have

$$
\begin{aligned}
& \left\|\left(\widehat{J}^{\lambda} \Phi^{\lambda}\right)(s, \varphi)-\left(\widehat{J}^{\lambda} \Psi^{\lambda}\right)(s, \varphi)\right\| \\
& \leq \int_{s}^{\infty}\left\|\bar{T}_{Q}(\tau, s)^{-1} Q(\tau)\right\| \\
& \cdot \mid f\left(\tau, x_{\tau}^{(\lambda, \widehat{\Phi}, \varphi)}, \Phi^{\lambda}\left(\tau, x_{\tau}^{(\lambda, \widehat{\Phi}, \varphi)}\right), \lambda\right) \\
& \quad-f\left(\tau, x_{\tau}^{(\lambda, \widehat{\Psi}, \varphi)}(\tau, \xi), \Psi^{\lambda}\left(\tau, x_{\tau}^{(\lambda, \widehat{\Psi}, \varphi)}\right), \lambda\right) \mid d \tau \\
& \leq\left[\frac{2 c K^{3} M}{(1-2 c K M)^{2}}+\frac{c K^{2}}{1-2 c K M}\right]\|\varphi\| \\
& \quad \cdot\|\widehat{\Phi}-\widehat{\Psi}\| \int_{s}^{\infty}\left[\frac{\mu(\tau)}{\mu(s)}\right]^{a-b} \nu(s)^{\varepsilon} \nu(\tau)^{\varepsilon-\gamma} d \tau \\
& \leq\left[\frac{2 c K^{3} M}{(1-2 c K M)^{2}}+\frac{c K^{2}}{1-2 c K M}\right] N\|\varphi\| \cdot\|\widehat{\Phi}-\widehat{\Psi}\| .
\end{aligned}
$$

By $\left(H_{8}\right)$ and $\left(H_{9}\right)$, we see that the operator $\hat{J}^{\lambda}: \mathscr{B} \rightarrow \mathscr{B}$ becomes a contraction. Therefore, given $\lambda \in Y$, for each $\varphi \in$ $E(s)$, there exists a unique function $\widehat{\Phi}=\Phi^{\lambda}$ satisfying (51). By (26) and Lemma 6, we obtain

$$
\begin{aligned}
\left\|\Omega_{\epsilon}^{\lambda}\left(s, \varphi, \Phi^{\lambda}(s, \varphi)\right)-\Omega_{\epsilon}\left(s, \psi, \Phi^{\lambda}(s, \psi)\right)\right\| \\
=\|\left(t, x_{t}^{(\lambda, \widehat{\Phi}, \varphi)}, \Phi^{\lambda}\left(t, x_{t}^{(\lambda, \widehat{\Phi}, \varphi)}\right)\right) \\
\quad-\left(t, x_{t}^{(\lambda, \widehat{\Phi}, \psi)}, \Phi^{\lambda}\left(t, x_{t}^{(\lambda, \widehat{\Phi}, \psi)}\right)\right) \| \\
\leq 2\left\|x_{t}^{(\lambda, \widehat{\Phi}, \varphi)}-x_{t}^{(\lambda, \widehat{\Phi}, \psi)}\right\| \\
\leq \frac{2 K}{1-2 c k M}\left[\frac{\mu(t)}{\mu(s)}\right]^{a} v(s)^{\varepsilon}\|\varphi-\psi\| .
\end{aligned}
$$

Finally, we turn to the proof of inequality (35) of the theorem. The following lemma is necessary for our establishing (35).

Lemma 10. Given $\varphi \in E(s)$, for each $\lambda, \eta \in Y$, one has

$$
\begin{aligned}
& \left\|x_{t}^{\left(\lambda, \Phi^{\lambda}, \varphi\right)}-x_{t}^{\left(\eta, \Phi^{\eta}, \varphi\right)}\right\| \\
& \quad \leq \frac{3 c K^{2} M}{(1-2 c K M)^{2}}\left[\frac{\mu(t)}{\mu(s)}\right]^{a} \nu(s)^{\varepsilon}\|\varphi\| \cdot|\lambda-\eta|,
\end{aligned}
$$

for every $s \geq 0, \varphi \in E(s)$.

Proof. By (26), (28), $\left(\mathrm{H}_{2}\right)$, and Lemma 5, we have

$$
\begin{aligned}
& q_{3}(\tau) \\
& :=\mid f\left(\tau, x_{\tau}^{\left(\lambda, \Phi^{\lambda}, \varphi\right)}, \Phi^{\lambda}\left(\tau, x_{\tau}^{\left(\lambda, \Phi^{\lambda}, \varphi\right)}\right), \lambda\right) \\
& -f\left(\tau, x_{\tau}^{\left(\eta, \Phi^{\eta}, \varphi\right)}, \Phi^{\eta}\left(\tau, x_{\tau}^{\left(\eta, \Phi^{\eta}, \varphi\right)}\right), \eta\right) \\
& \leq \mid f\left(\tau, x_{\tau}^{\left(\lambda, \Phi^{\lambda}, \varphi\right)}, \Phi^{\lambda}\left(\tau, x_{\tau}^{\left(\lambda, \Phi^{\lambda}, \varphi\right)}\right), \lambda\right) \\
& -f\left(\tau, x_{\tau}^{\left(\lambda, \Phi^{\lambda}, \varphi\right)}, \Phi^{\lambda}\left(\tau, x_{\tau}^{\left(\lambda, \Phi^{\lambda}, \varphi\right)}\right), \eta\right) \mid \\
& +\mid f\left(\tau, x_{\tau}^{\left(\lambda, \Phi^{\lambda}, \varphi\right)}, \Phi^{\lambda}\left(\tau, x_{\tau}^{\left(\lambda, \Phi^{\lambda}, \varphi\right)}\right), \eta\right) \\
& -f\left(\tau, x_{\tau}^{\left(\eta, \Phi^{\eta}, \varphi\right)}, \Phi^{\eta}\left(\tau, x_{\tau}^{\left(\eta, \Phi^{\eta}, \varphi\right)}\right), \eta\right) \\
& \leq c \nu(\tau)^{-\gamma}\left[2\left\|x_{\tau}^{\left(\lambda, \Phi^{\lambda}, \varphi\right)}\right\| \cdot|\lambda-\eta|\right. \\
& +\left\|x_{\tau}^{\left(\lambda, \Phi^{\lambda}, \varphi\right)}-x_{\tau}^{\left(\eta, \Phi^{\eta}, \varphi\right)}\right\| \\
& \left.+\left\|\Phi^{\lambda}\left(\tau, x_{\tau}^{\left(\lambda, \Phi^{\lambda}, \varphi\right)}\right)-\Phi^{\eta}\left(\tau, x_{\tau}^{\left(\eta, \Phi^{\eta}, \varphi\right)}\right)\right\|\right] \\
& \leq c \nu(\tau)^{-\gamma}\left[3\left\|x_{\tau}^{\left(\lambda, \Phi^{\lambda}, \varphi\right)}\right\| \cdot\|\varphi\| \cdot|\lambda-\eta|\right. \\
& \left.+2\left\|x_{\tau}^{\left(\lambda, \Phi^{\lambda}, \varphi\right)}-x_{\tau}^{\left(\eta, \Phi^{\eta}, \varphi\right)}\right\|\right] \\
& \leq c \nu(\tau)^{-\gamma}\left\{\frac{3 K}{1-2 c K M}\left[\frac{\mu(\tau)}{\mu(s)}\right]^{a} \nu(s)^{\varepsilon}\|\varphi\|\right. \\
& \left.\cdot|\lambda-\eta|+2\left\|x_{\tau}^{\left(\lambda, \Phi^{\lambda}, \varphi\right)}-x_{\tau}^{\left(\eta, \Phi^{\eta}, \varphi\right)}\right\|\right\} .
\end{aligned}
$$


Thus, it follows from (24) and (65) that

$$
\begin{aligned}
\left\|x_{\tau}^{\left(\lambda, \Phi^{\lambda}, \varphi\right)}-x_{\tau}^{\left(\eta, \Phi^{\eta}, \varphi\right)}\right\| & \int_{s}^{t}\|\bar{T}(t, \tau) P(\tau)\| q_{3}(\tau) d \tau \\
\leq & \frac{3 c K^{2}}{1-2 c K M}\left[\frac{\mu(t)}{\mu(s)}\right]^{a} \nu(s)^{\varepsilon}\|\varphi\| \\
& \cdot|\lambda-\eta| \int_{s}^{t} \nu(\tau)^{\varepsilon-\gamma} d \tau+2 c K\left[\frac{\mu(t)}{\mu(s)}\right]^{a} \nu(s)^{\varepsilon}\|\varphi\| \\
& \left.\cdot\left\|x^{\left(\lambda, \Phi^{\lambda}, \varphi\right)}-x^{\left(\eta, \Phi^{\eta}, \varphi\right)}\right\|^{\prime}\right]_{s}^{t} \nu(\tau)^{\varepsilon-\gamma} d \tau \\
\leq & \frac{3 c K^{2} M}{1-2 c K M}\left[\frac{\mu(t)}{\mu(s)}\right]^{a} \nu(s)^{\varepsilon}\|\varphi\| \\
& \cdot|\lambda-\eta|+2 c K M\left[\frac{\mu(t)}{\mu(s)}\right]^{a} \nu(s)^{\varepsilon}\|\varphi\| \\
& \cdot\left\|x^{\left(\lambda, \Phi^{\lambda}, \varphi\right)}-x^{\left(\eta, \Phi^{\eta}, \varphi\right)}\right\|^{\prime} \cdot
\end{aligned}
$$

This proves that inequality (64) holds.

Therefore, by (28) and Lemma 10, we get

$$
\begin{gathered}
\left\|\Omega_{\epsilon}^{\lambda}\left(s, \varphi, \Phi^{\lambda}(s, \varphi)\right)-\Omega_{\epsilon}^{\eta}\left(s, \varphi, \Phi^{\eta}(s, \varphi)\right)\right\| \\
=\|\left(t, x_{t}^{\left(\lambda, \Phi^{\lambda}, \varphi\right)}, \Phi^{\lambda}\left(t, x_{t}^{\left(\lambda, \Phi^{\lambda}, \varphi\right)}\right)\right) \\
\quad-\left(t, x_{t}^{\left(\eta, \Phi^{\eta}, \varphi\right)}, \Phi^{\lambda}\left(t, x_{t}^{\left(\lambda, \Phi^{\eta}, \varphi\right)}\right)\right) \| \\
\leq 2\left\|x_{t}^{\left(\lambda, \Phi^{\lambda}, \varphi\right)}-x_{t}^{\left(\eta, \Phi^{\eta}, \varphi\right)}\right\| \\
+\left\|x_{t}^{\left(\lambda, \Phi^{\lambda}, \varphi\right)}\right\| \cdot|\lambda-\eta|
\end{gathered}
$$

$$
\begin{aligned}
\leq & {\left[\frac{6 c K^{2} M}{(1-2 c K M)^{2}}+\frac{K}{1-2 c K M}\right]\left[\frac{\mu(t)}{\mu(s)}\right]^{a} \nu(s)^{\varepsilon}\|\varphi\| } \\
& \cdot|\lambda-\eta| .
\end{aligned}
$$

This completes the proof of Theorem 4 .

\section{Example}

In this section, we provide an example to demonstrate the derived results. Consider the delay system

$$
\begin{aligned}
x^{\prime}= & {\left[-\frac{4}{3}+\frac{2 t}{3\left(1+t^{2}\right)}\right.} \\
& \left.\times\left(\log \left(1+t^{2}\right) \cos \left(\log \left(1+t^{2}\right)\right)-1\right)\right] x \\
& +\widehat{c}\left(1+t^{2}\right)^{-\gamma}\left(\lambda_{1}+\sin t\right) x(t-1), \\
y^{\prime}= & \frac{5}{3}+\frac{2 t}{3\left(1+t^{2}\right)} \\
& \left.\times\left(\log \left(1+t^{2}\right) \cos \left(\log \left(1+t^{2}\right)\right)-1\right)\right] y \\
& +\widehat{c}\left(1+t^{2}\right)^{-\gamma}\left(\lambda_{2}+\cos t\right) x(t-1),
\end{aligned}
$$

where $\mathscr{B}=C\left([-1,0], R^{2}\right)$ and $\lambda=\left(\lambda_{1}, \lambda_{2}\right)=[-1,1] \times[-1,1]$. Then, we have

$$
\begin{aligned}
& f(t, \varphi, \lambda) \\
& =\left(\begin{array}{cc}
\widehat{c}\left(1+t^{2}\right)^{-\gamma}\left(\lambda_{1}+\sin t\right) & 0 \\
0 & \widehat{c}\left(1+t^{2}\right)^{-\gamma}\left(\lambda_{2}+\cos t\right)
\end{array}\right) \\
& \quad \times \varphi(-1) .
\end{aligned}
$$

Setting $P(t)(x, y)=x$ and $Q(t)(x, y)=y$, we have

$$
=\left(\frac{e^{t}}{e^{s}}\right)^{-4 / 3} e^{(1 / 3)\left[\log \left(1+t^{2}\right) \sin \left(\log \left(1+t^{2}\right)-1\right)+\cos \left(\log \left(1+t^{2}\right)\right)-\cos \left(\log \left(1+s^{2}\right)\right)-\log \left(1+s^{2}\right)\left(\sin \left(\log \left(1+s^{2}\right)\right)-1\right)\right]},
$$

$T(t, s) Q(s)$

$$
=\left(\frac{e^{t}}{e^{s}}\right)^{5 / 3} e^{(1 / 3)\left[\log \left(1+t^{2}\right) \sin \left(\log \left(1+t^{2}\right)-1\right)+\cos \left(\log \left(1+t^{2}\right)\right)-\cos \left(\log \left(1+s^{2}\right)\right)-\log \left(1+s^{2}\right)\left(\sin \left(\log \left(1+s^{2}\right)\right)-1\right)\right]}
$$


which implies that

$$
\begin{aligned}
& \|T(t, s) P(s)\| \leq e^{2 / 3}\left(\frac{e^{t}}{e^{s}}\right)^{-4 / 3}\left(1+s^{2}\right)^{2 / 3}, \\
& \left\|T(t, s)^{-1} Q(s)\right\| \leq e^{2 / 3}\left(\frac{e^{t}}{e^{s}}\right)^{-5 / 3}\left(1+t^{2}\right)^{2 / 3} .
\end{aligned}
$$

This shows that the linear part of (68) admits a nonuniform $(\mu, v)$-dichotomy with

$$
\begin{gathered}
\mu(t)=e^{t}, \quad v(t)=1+t^{2}, \quad K=e^{2 / 3}, \\
a=-\frac{4}{3}, \quad b=\frac{5}{3}, \quad \varepsilon=\frac{2}{3} .
\end{gathered}
$$

Furthermore, we see that $f(t, 0, \lambda)=0$ and

$$
\begin{gathered}
|f(t, \varphi, \lambda)-f(t, \psi, \lambda)| \leq 2 \widehat{c}\left(1+t^{2}\right)^{-\gamma}\|\varphi-\psi\|, \\
|f(t, \varphi, \lambda)-f(t, \varphi, \eta)| \leq \widehat{c}\left(1+t^{2}\right)^{-\gamma}\|\varphi\| \cdot|\lambda-\eta| .
\end{gathered}
$$

Taking suitable $\gamma>0$ and sufficiently small $c=2 \widehat{c}$, we conclude that (68) has a stable invariant manifold provided that $\left(H_{1}\right)-\left(H_{3}\right)$ and $\left(H_{5}\right)-\left(H_{9}\right)$ hold.

\section{Conflict of Interests}

The author declares that there is no conflict of interests regarding the publishing of this paper.

\section{References}

[1] O. Perron, "Die Stabilitätsfrage bei Differentialgleichungen," Mathematische Zeitschrift, vol. 32, no. 1, pp. 703-728, 1930.

[2] R. J. Sacker and G. R. Sell, "Existence of dichotomies and invariant splittings for linear differential systems. I," Journal of Differential Equations, vol. 15, pp. 429-458, 1974.

[3] R. J. Sacker and G. R. Sell, "Existence of dichotomies and invariant splittings for linear differential systems. II," Journal of Differential Equations, vol. 22, no. 2, pp. 478-496, 1976.

[4] R. J. Sacker and G. R. Sell, "Existence of dichotomies and invariant splittings for linear differential systems. III," Journal of Differential Equations, vol. 22, no. 2, pp. 497-522, 1976.

[5] R. J. Sacker and G. R. Sell, "Dichotomies for linear evolutionary equations in Banach spaces," Journal of Differential Equations, vol. 113, no. 1, pp. 17-67, 1994.

[6] L. Barreira and C. Valls, Stability of Nonautonomous Differential Equations, vol. 1926 of Lecture Notes in Mathematics, Springer, Berlin, Germany, 2008.

[7] L. Barreira and Y. B. Pesin, Lyapunov Exponents and Smooth Ergodic Theory, vol. 23 of University Lecture Series, American Mathematical Society, Providence, RI, Providence, RI, USA, 2002.

[8] J. Massera and J. Schäfer, Linear Differential Equations and Function Spaces, vol. 21 of Pure and Applied Mathematics, Academic Press, 1966.

[9] J. L. Dalec/kiĭ and M. G. Kreĭn, Stability of Solutions of Differential Equations in Banach Space, vol. 43, American Mathematical Society, 1974.
[10] W. A. Coppel, Dichotomies in Stability Theory, Springer, 1978.

[11] J. L. Massera and J. J. Schäffer, "Linear differential equations and functional analysis. I," Annals of Mathematics, vol. 67, no. 3, pp. 517-573, 1958.

[12] L. Barreira and C. Valls, "Stable manifolds for nonautonomous equations without exponential dichotomy," Journal of Differential Equations, vol. 221, no. 1, pp. 58-90, 2006.

[13] L. Barreira and C. Valls, "Parameter dependence of stable manifolds under nonuniform hyperbolicity," Journal of Mathematical Analysis and Applications, vol. 358, no. 2, pp. 419-426, 2009.

[14] S.-N. Chow and H. Leiva, "Existence and roughness of the exponential dichotomy for skew-product semiflow in Banach spaces," Journal of Differential Equations, vol. 120, no. 2, pp. 429477, 1995.

[15] V. A. Pliss and G. R. Sell, "Robustness of exponential dichotomies in infinite-dimensional dynamical systems," Journal of Dynamics and Differential Equations, vol. 11, no. 3, pp. 471513, 1999.

[16] A. J. G. Bento and C. M. Silva, "Generalized nonuniform dichotomies and local stable manifolds," Journal of Dynamics and Differential Equations, vol. 25, no. 4, pp. 1139-1158, 2013.

[17] L. Barreira and C. Valls, "Polynomial growth rates," Nonlinear Analysis: Theory, Methods \& Applications, vol. 71, no. 11, pp. 5208-5219, 2009.

[18] L. Barreira, M. Fan, C. Valls, and J. Zhang, "Robustness of nonuniform polynomial dichotomies for difference equations," Topological Methods in Nonlinear Analysis, vol. 37, no. 2, pp. 357376, 2011.

[19] A. J. Bento and C. Silva, "Stable manifolds for nonuniform polynomial dichotomies," Journal of Functional Analysis, vol. 257, no. 1, pp. 122-148, 2009.

[20] L. Barreira and C. Valls, "Growth rates and nonuniform hyperbolicity," Discrete and Continuous Dynamical Systems, vol. 22, no. 3, pp. 509-528, 2008.

[21] L. Barreira and C. Valls, "Robustness of general dichotomies," Journal of Functional Analysis, vol. 257, no. 2, pp. 464-484, 2009.

[22] L. Barreira and C. Valls, "Stable invariant manifolds for parabolic dynamics," Journal of Functional Analysis, vol. 257, no. 4, pp. 1018-1029, 2009.

[23] X. Chang, J. Zhang, and J. Qin, "Robustness of nonuniform $(\mu, v)$-dichotomies in Banach spaces," Journal of Mathematical Analysis and Applications, vol. 387, no. 2, pp. 582-594, 2012.

[24] A. Bento and C. Silva, "Nonuniform $(\mu, v)$-dichotomies and local dynamics of difference equations," Journal of Dynamics and Differential Equations, vol. 25, pp. 1139-1158, 2013.

[25] J. K. Hale and S. M. V. Lunel, Introduction to Functional Differential Equations, Springer, Berlin, Germany, 1993.

[26] L. Barreira, M. Fan, C. Valls, and J. Zhang, "Parameter dependence of stable manifolds for delay equations with polynomial dichotomies," Journal of Dynamics and Differential Equations, vol. 24, no. 1, pp. 101-118, 2012. 


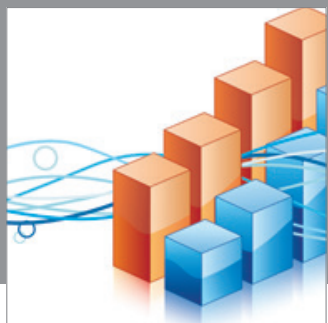

Advances in

Operations Research

mansans

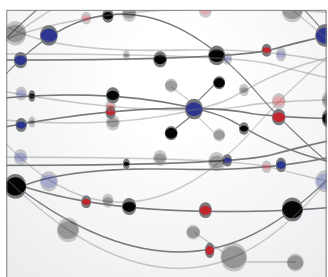

The Scientific World Journal
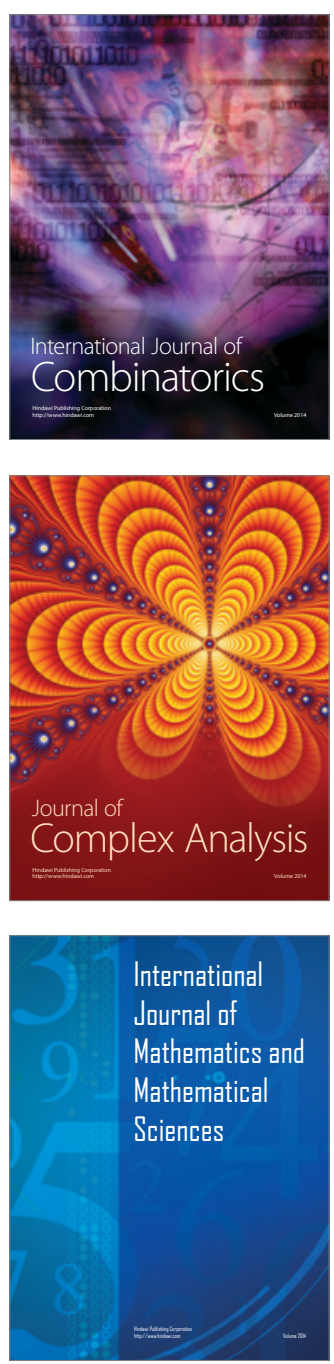
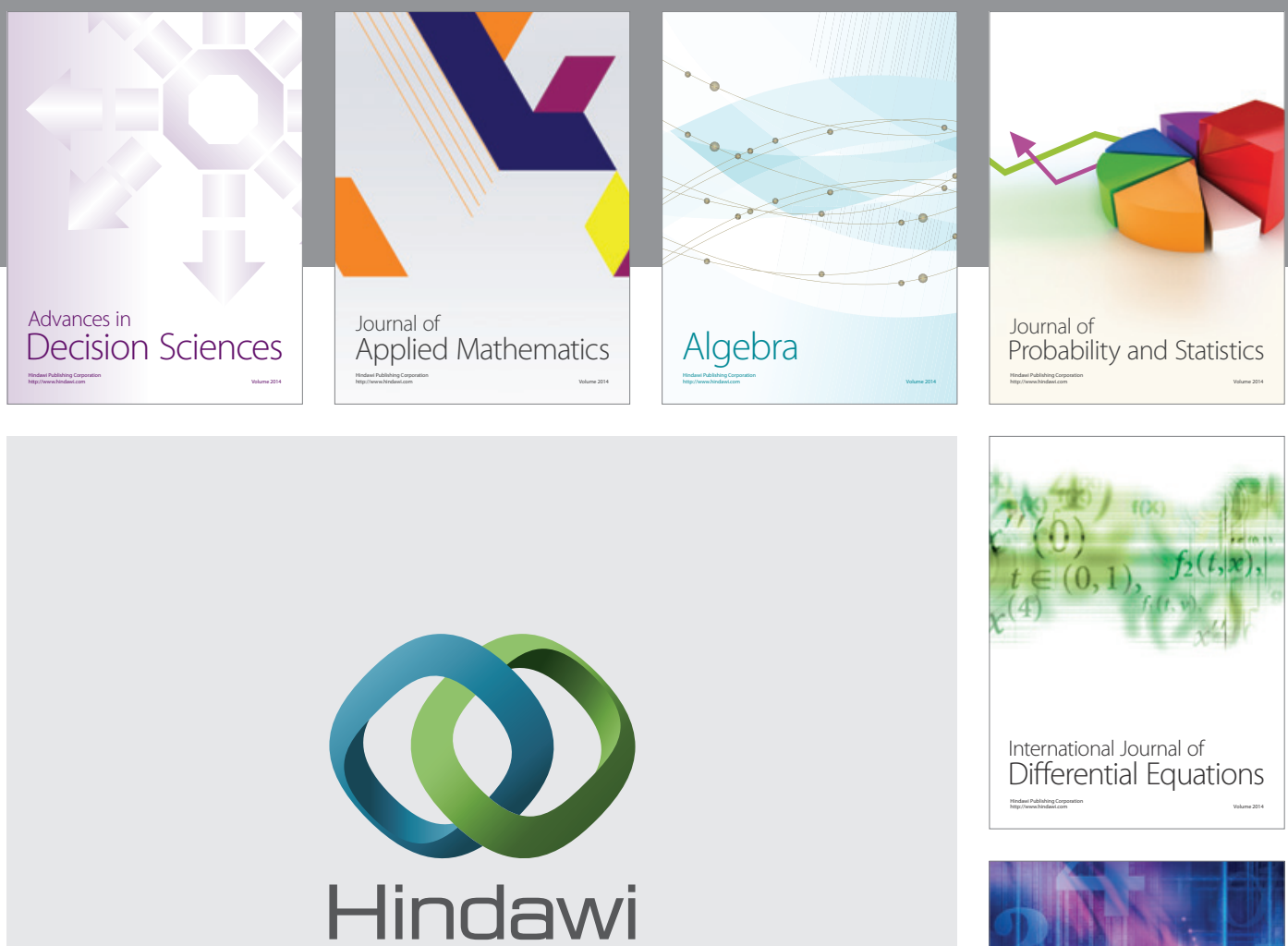

Submit your manuscripts at http://www.hindawi.com
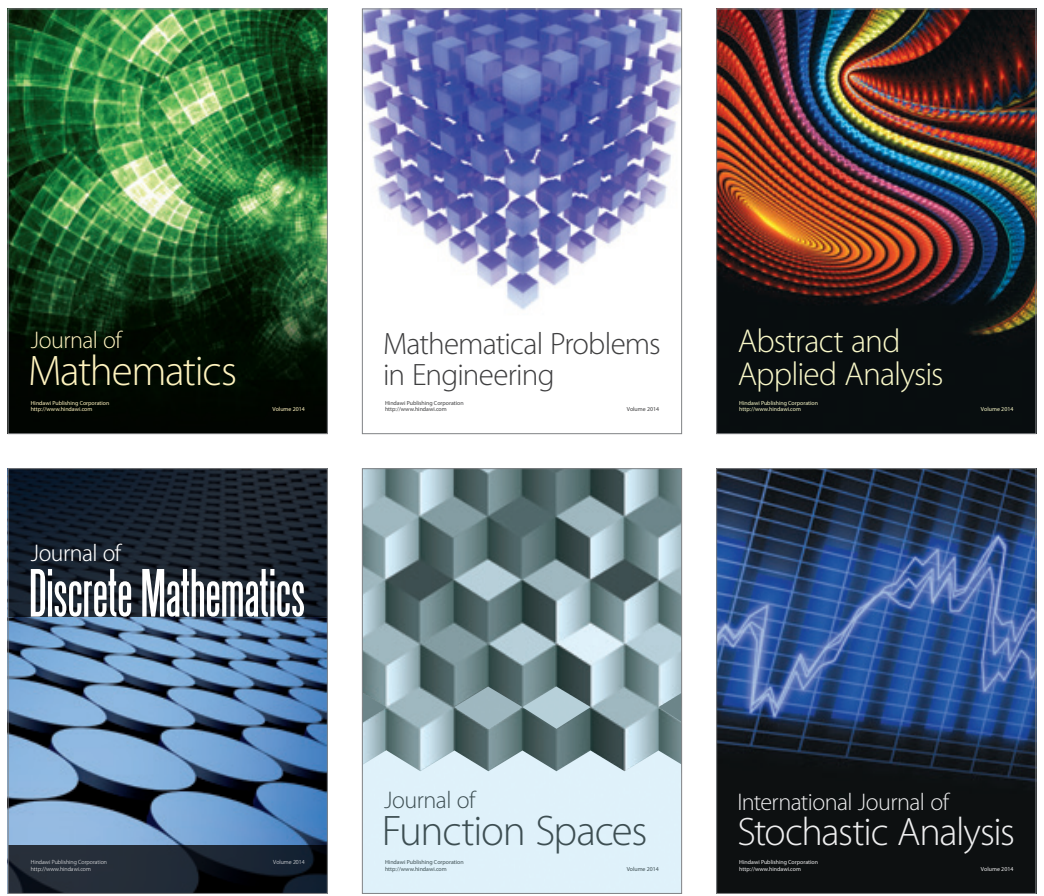

Journal of

Function Spaces

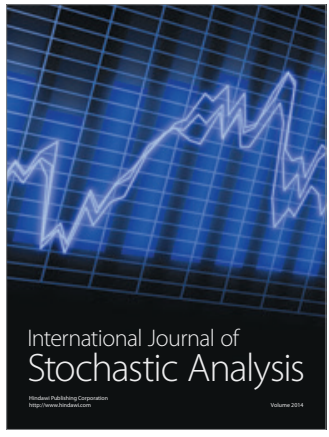

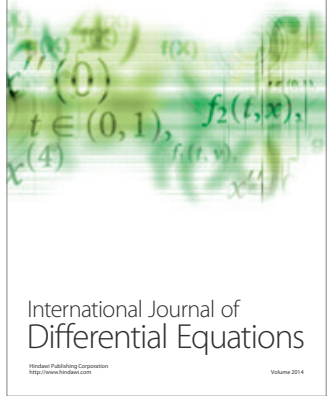
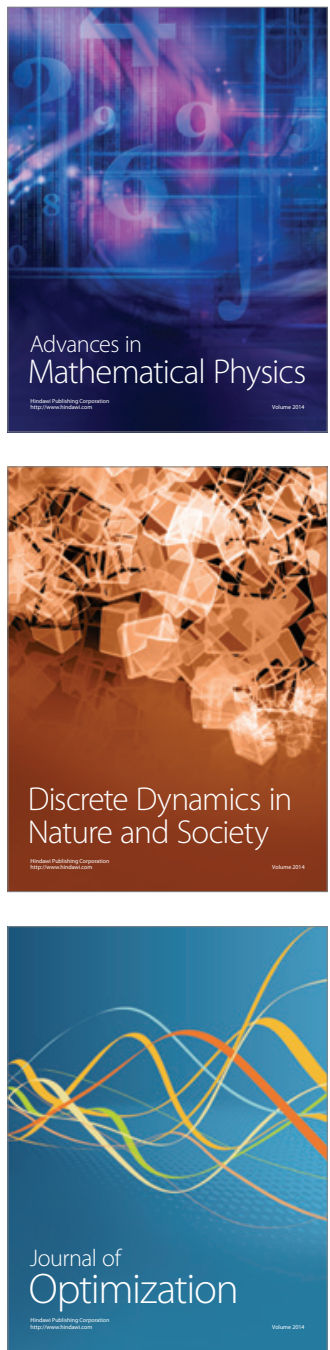